\title{
TOPOLOGICAL CONJUGACY AND STRUCTURAL STABILITY FOR DISCRETE DYNAMICAL SYSTEMS ${ }^{1}$
}

\author{
BY J. W. ROBBIN
}

\begin{abstract}
Topological conjugacy and various concepts of structural stability are defined, motivated, and criticized. Two basic problems emerge: characterization of structural stability and classification up to topological conjugacy. Solutions to these problems are outlined for linear automorphisms and the general characterization problem is discussed.
\end{abstract}

This paper is an expanded version of an invited address given at the summer meeting of the American Mathematical Society in September, 1971. Much of the material here was also covered in a series of lectures given at the Institut des Hautes Etudes Scientifiques in the fall of 1971.

Our notation is that of [2] and [3]. $M$ always denotes a smooth $\left(C^{\infty}\right)$, finite-dimensional manifold, sometimes but not always compact, and always without boundary. $T M$ denotes the tangent bundle of $M$ and for $x \in M, T_{x} M$ denotes the tangent space to $M$ at $x$. If $h: M \rightarrow M^{\prime}$ is a $C^{1}$ map, then $T h: T M \rightarrow T M^{\prime}$ denotes the tangent map of $h$ and for $x \in M$, $T_{x} h: T_{x} M \rightarrow T_{h(x)} M^{\prime}$ denotes the tangent (= derivative) to $h$ at $x$. Thus if $\dot{x} \in T_{x} M$ is the tangent vector at 0 to a curve $c: R \rightarrow M$ with $c(0)=x$ then $\left(T_{x} h\right) \dot{x}$ is the tangent vector at 0 to the curve $h \circ c: R \rightarrow M^{\prime} . R$ always denotes the (additive group of) real numbers and $\boldsymbol{Z}$ the (additive group of) integers, and $\boldsymbol{C}$ denotes the complex numbers. By a diffeomorphism we always mean a $C^{1}$ diffeomorphism; i.e. a map $h: M \rightarrow M^{\prime}$ which is bijective and such that both $h$ and $h^{-1}$ are $C^{1}$. We denote by $\operatorname{Diff}(M)$ the group of all $C^{1}$ diffeomorphisms of $M$ (i.e. from $M$ onto $M$ ); the group operation is, of course, composition.

An expanded version of an invited address given at the summer meeting of the American Mathematical Society in September 1971; received by the editors March 27, 1972.

AMS 1970 subject classifications. Primary 58F00; Secondary 58F10, 58F15.

Key words and phrases. Cascade, flow, topological conjugacy, differentiable conjugacy, structural stability, north pole-south pole map, hyperbolic toral automorphism, strong structural stability, absolute structural stability, relative structural stability, symplectic manifold, twist stability, topological stability, semistability, Yin-Yang problem, hyperbolic linear automorphism, in-set, out-set, on-set, Anosov diffeomorphism, selector, conjugacy selector, adjoint representation, ergodic, infinitesimally ergodic, Sobolev space, hyperbolic invariant set, nonwandering set, $\Omega$-hyperbolic, $P$-hyperbolic, axiom A, weak axiom A, essential spectrum, strong transversality condition, weak transversality condition, in-set, stable manifold, out-set, unstable manifold.

${ }^{1}$ AcKNowledgement. Research supported by the National Science Foundation (contract 144-B695), the Wisconsin Alumni Research Foundation (project 120432), and L'Institut des Hautes Etudes Scientifiques. 
The paper begins with a long introduction (\$1) where the basic definitions and problems are defined and motivated. I have tried there to explain how the concept of structural stability might be useful in practical applications and I have given several definitions of the concept and indicated some of their weaknesses. The two basic problems defined there (Problems I and II of $§ 1 . E$ ) are those of characterizing structural stability and classifying dynamical systems up to topological conjugacy. In $\$ \S 2$ and $3 \mathrm{I}$ indicate solutions of these two problems for the case of linear automorphisms; the bulk of the rest of the paper is devoted to the problem of characterizing structural stability on an arbitrary compact manifold.

During the preparation of this paper I was supported by the National Science Foundation (contract 144-B695), the Wisconsin Alumni Research Foundation (project 120432), and the I.H.E.S., and I would like to express my gratitude to this support. I would also like to thank the I.H.E.S. and its director N. Kuiper for their hospitality. Thanks also to R. Abraham for reading and criticizing a first draft of the introduction.

The present paper develops some of the ideas in the important survey article of Smale [51]; the interested reader will doubtless want to consult this reference.

\section{Introduction.}

1A. The concept of a dynamical system. In physics, a dynamical system is usually described by an ordinary differential equation; i.e. a vector field on a manifold $M$. Under suitable hypotheses the vector field generates a "flow" on the manifold; i.e. a group homomorphism

$$
\boldsymbol{R} \rightarrow \operatorname{Diff}(M): t \rightarrow f^{t} .
$$

The study of the topological properties of such flows is called "the qualitative theory of differential equations".

The discrete analog of a flow is a group homomorphism

$$
Z \rightarrow \operatorname{Diff}(M): n \rightarrow f^{n} .
$$

Such a group homomorphism is called a "cascade" in [5]; it is completely determined by its generator $f$. This generator is the discrete analog of the "infinitesimal generator" (i.e. the vector field) of a flow. Thus for us a discrete dynamical system on a manifold $M$ is simply a $C^{1}$ diffeomorphism $f$ from $M$ onto itself; i.e. an element of $\operatorname{Diff}(M)$.

We shall give $\operatorname{Diff}(M)$ the $C^{1}$ topology (the $C^{1}$ fine topology if $M$ is not compact: see [26]). With this topology, $\operatorname{Diff}(M)$ is an open subset of $C^{1}(M, M)$ (see [26]).

The starting point for a topological theory of discrete dynamical systems must be a notion of "topological equivalence" of two systems. 
1.1. Definition. Let $M$ and $M^{\prime}$ be smooth manifolds and $f \in \operatorname{Diff}(M)$ and $g \in \operatorname{Diff}\left(M^{\prime}\right)$. Then $f$ and $g$ are topologically conjugate iff there exists a homeomorphism $\varphi: M \rightarrow M^{\prime}$ such that $g=\varphi \circ f \circ \varphi^{-1}$. Such a homeomorphism $\varphi$ is called a topological conjugacy between $f$ and $g$.

We shall also say that $f$ and $g$ are differentiably conjugate and that $\varphi$ is a differentiable conjugacy between $f$ and $g$ in case $\varphi$ satisfying 1.1 is a diffeomorphism. Ho vever, we shall soon see that this notion is far too strong for stability problems. Thus the word "conjugate" unmodified shall always mean "topologically conjugate".

Note that if $\varphi$ is a conjugacy between $f$ and $g$, then it is also a conjugacy between $f^{n}$ and $g^{n}$ for $n \in Z$ :

$$
g^{n}=\varphi \circ f^{n} \circ \varphi^{-1}
$$

Thus, in particular, $\varphi$ maps periodic points of $f$ to periodic points of $g$. We might expect that the appropriate equivalence relations for flows $f^{t}$ and $g^{t}$ should be

$$
g^{t}=\varphi \circ f^{t} \circ \varphi^{-1}
$$

for $t \in \boldsymbol{R}$. This will not do; if we reparameterize a flow $f^{t}$ by slowing it down a tiny bit to obtain a flow $g^{t}$, then $f^{t}$ and $g^{t}$ will not be equivalent in the sense of (2) as the periods of periodic orbits have changed. Nonetheless, the reparameterization has not changed the qualitative picture in any essential way. Thus a good definition of equivalence for flows must be more complicated than (2); this is why the discrete theory is much simpler than the continuous theory. Many different definitions of equivalence for flows have been proposed (see [42]). Of course, (2) could be a reasonable definition for flows without periodic orbits; e.g. gradient flows.

If $f$ and $g$ are topologically conjugate, then they have exactly the same topological properties; conversely, to show that particular $f$ and $g$ are not conjugate, one exhibits a topological property of $f$ which fails for $g$. We illustrate this with some simple examples where $M=M^{\prime}=\boldsymbol{R}$.

1.2. EXAMPLE. $f(x)=2 x, g(x)=8 x$ for $x \in R$. $f$ and $g$ are conjugate; a conjugacy $\varphi: R \rightarrow R$ is given by $\varphi(x)=x^{3}$ for $x \in R$. Note that $f$ and $g$ are not differentiably conjugate, for if $\varphi(2 x)=8 \varphi(x)$ and $\varphi$ is differentiable, then $\varphi(0)=0$ and $D \varphi(0)=0$.

1.3. ExAmple. $f(x)=2 x, g(x)=-2 x$. $f$ and $g$ are not conjugate as $f$ preserves orientation and $g$ reverses orientation.

1.4. EXAMPLE. $f(x)=2 x, g(x)=\frac{1}{2} x$. $f$ and $g$ are not conjugate as $\lim _{n \rightarrow \infty} g^{n}(x)=0 \in \boldsymbol{R}$ for all $x \in \boldsymbol{R}$ while $\lim _{n \rightarrow \infty} f^{n}(x)=\infty \notin \boldsymbol{R}$ for $x \neq 0$.

1.5. ExAmple. $f(x)=a x, g(x)=b x$ where $a, b \in \boldsymbol{R}\{0,1,-1\}$. Combining the ideas of the previous examples we see that $f$ and $g$ are conjugate 
if and only if $a$ and $b$ belong to the same component of $\boldsymbol{R} \backslash\{0,1,-1\}$. When this is so a conjugacy $\varphi$ between $f$ and $g$ is given by $\varphi(x)=x^{c}$ where $|a|^{c}=|b|$. In $\S 3$ we will generalize this example from $\boldsymbol{R}$ to $\boldsymbol{R}^{\boldsymbol{m}}$.

1B. Structural stability. We now turn to the notion of structural stability which is motivated by the following metaphysical ${ }^{2}$ problem: Suppose a physicist has a physical theory which assigns to each physical system $S$ of a certain type a manifold $M$ whose points are to represent the possible states of $S$ and a dynamical system $g \in \operatorname{Diff}(M)$ which describes the evolution of $S$ in (discrete) time. The system $g$ will depend on certain parameters (e.g. masses of particles) which must be determined by measurements subject to experimental error. Also the theory may only be an approximation to a better theory (e.g. classical approximation to a more exact relativistic theory) and this also introduces error.

Given a physical system $S$ our physicist performs the measurements and applies the theory to obtain a dynamical system $f$, but because of the error mentioned above, all he (or she) knows about the "true system" $g$ (the one which accurately describes $S$ ) is that it is close to $f$; i.e. $g$ lies in a certain neighborhood $N$ of $f$ where the size of $N$ is determined by the error.

The physicist will make predictions of the qualitative behavior of the system $S$ by studying $f$, but the system is actually described by $g$ which he does not (and cannot) know. His predictions are worthless if $g$ is radically different from $f$. He would therefore be happy to know that $g$ is conjugate to $f$. This will be true if $f$ is structurally stable and the error neighborhood $N$ is sufficiently small.

1.6. Definition. Let $M$ be a smooth manifold and $f \in \operatorname{Diff}(M)$. Then $f$ is structurally stable iff there exists a neighborhood $N$ of $f$ in $\operatorname{Diff}(M)$ such that every $g \in N$ is topologically conjugate to $f$.

To the best of my knowledge, this definition (or rather an analogous one for flows) is due to Andronov and Pontrjagin [4].

We shall now consider some examples. The fundamental intuition is this: A system is structurally stable iff its qualitative behavior is unchanged by a small perturbation.

1.7. EXAMPLE. Let $f$ rotate the two-sphere $S^{2}$ through angle $\theta=2 \pi / 3$. Thus $f^{3}=$ identity. If $g$ rotates $S^{2}$ through angle $\theta+\varepsilon$, then $g^{3} \neq$ id. Hence $f$ is not structurally stable. Similar considerations show that no rotation of a sphere is structurally stable.

1.8. EXAMPLE. Identify the two-sphere $S^{2}$ with the extended complex plane: $S^{2}=C \cup\{\infty\}$. Let $f: S^{2} \rightarrow S^{2}$ be

${ }^{2}$ The word metaphysical is here taken in its most literal sense. 


$$
\begin{aligned}
& f(z)=2 z, \quad z \in C, \\
& f(\infty)=\infty .
\end{aligned}
$$

This diffeomorphism is called the "north pole-south pole map"; if 0 is the "north pole" and $\infty$ is the "south pole", then the poles are the only fixed points and every point not a pole tends to the south pole under positive iteration and to the north pole under negative iteration; i.e.

$$
\lim _{n \rightarrow \infty} f^{n}(x)=\infty, \quad \lim _{n \rightarrow \infty} f^{-n}(x)=0,
$$

for $x \neq 0, \infty$. See Figure 1 .

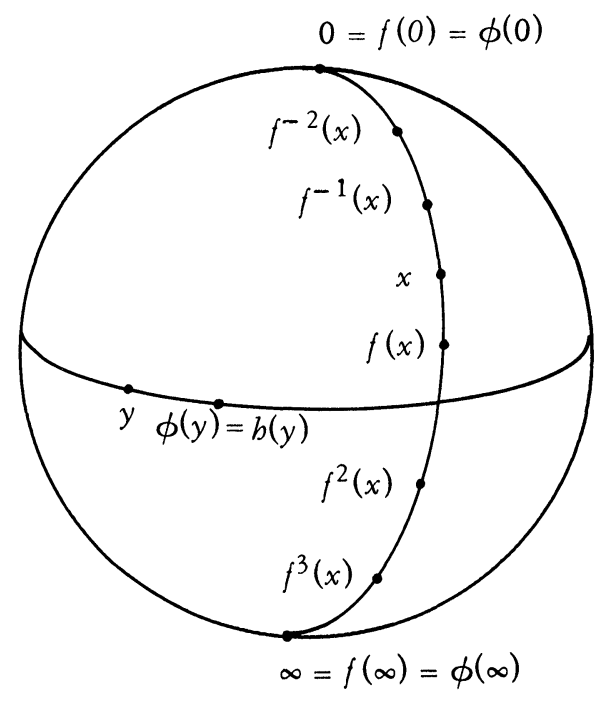

FIGURE 1. The north pole-south pole map on $S^{2}$.

The north pole-south pole map is structurally stable. This follows from the general theorem described below $(\S 9)$ but the reader may want to amuse himself by finding a direct proof.

This example illustrates an important technical difficulty in proving structural stability; Conjugacies between $f$ and a perturbation $g$ of $f$ need not be unique. To illustrate this take $f=g$. Let $h: S^{1} \rightarrow S^{1}$ be continuous and "suspend" $h$ to obtain $\varphi: S^{2} \rightarrow S^{2}$ :

$$
\varphi(z)=\operatorname{rh}\left(e^{i \theta}\right), \quad \varphi(\infty)=\infty,
$$

for $z=r e^{i \theta} \in C$. Then $f \circ \varphi=\varphi \circ f$ and $\varphi$ is a homeomorphism if and only if $h$ is.. In particular, for $g$ close to $f$ we can find solutions $\varphi$ of $g \circ \varphi=$ 
$\varphi \circ f$ which are close to the identity and not homeomorphisms. To prove structural stability one usually "solves" for $\varphi$ in this equation $g \circ \varphi=\varphi \circ f$ and then shows that $\varphi$ is a homeomorphism. The example shows that one must be careful about the particular solution $\varphi$ which one takes.

1.9. EXAMPLE. A continuous group automorphism of the torus $T^{m}=$ $\boldsymbol{R}^{m} / \boldsymbol{Z}^{m}$ is described by an $m \times m$ integer matrix with determinant \pm 1 . If this matrix has no eigenvalue of complex modulus 1 , then the automorphism is called a "hyperbolic toral automorphism". The periodic points of such an automorphism are dense; indeed any point with rational coordinates is periodic. The simplest example is found by taking the matrix $\left(\begin{array}{ll}2 & 1 \\ 1 & 1\end{array}\right)$.

Hyperbolic toral automorphisms are examples of Anosov diffeomorphisms and by the theorem of Anosov (see $\S 4$ ) they are structurally stable. In contrast to Example 1.8, the conjugacy $\varphi$ is unique (provided that it is sufficiently close to the identity).

1C. Quantitative problems. Suppose as before that a physicist is studying a physical system $S$ whose possible states are represented by the points of a smooth manifold $M$ and with dynamics $g \in \operatorname{Diff}(M)$. Thus if $y \in M$ represents the state of the system $S$ at time $t=0$, then $g^{n}(y)$ represents the state of the system at time $t=n$. As before, the physicist measures $g$ and obtains $f \in \operatorname{Diff}(M)$ and an error neighborhood $N$ of $f$ (so that $g \in N$ ). If he wants to make quantitative (as well as qualitative) predictions based on $f$, he will have to know not only that $g$ is conjugate to $f$ but also how far the conjugacy $\varphi$ between $f$ and $g$ is from the identity. (We will illustrate this below.) This motivates the following:

1.10. Definition. ${ }^{3} f \in \operatorname{Diff}(M)$ is strongly structurally stable iff for every neighborhood $N_{0}$ of the identity in $C^{0}(M, M)$ there is a neighborhood $N$ of $f$ in $\operatorname{Diff}(M)$ such that for all $g \in N$ there exists a homeomorphism $\varphi \in N_{0}$ which is a conjugacy between $f$ and $g$.

(The appropriate topology on $C^{0}(M, M)$ is the compact-open topology which reduces to the $C^{0}$-topology when $M$ is compact.)

We next give an example to show how our physicist might use this definition.

Let $M$ be compact and $d$ be a metric on $M$. This determines a metric $d_{0}$ on $C^{0}(M, M)$ by

for $\varphi, \psi \in C^{0}(M, M)$.

$$
d_{0}(\varphi, \psi)=\sup _{x \in M} d(\varphi(x), \psi(x))
$$

Suppose the measured system $f$ has a fixed point $p \in M$ with open basin

\footnotetext{
${ }^{3}$ It is reasonable to conjecture that strong structural stability is equivalent to structural stability; there is no known counterexample to this conjecture.
} 
of attraction $U$; i.e., $\lim _{n \rightarrow \infty} f^{n}(x)=p$ for $x \in U$. If the true system $g$ is conjugate to $f$, then $g$ will have a fixed point $q$ with $q=\varphi(p)$ having open basin of attraction $V=\varphi(U)$. Here $\varphi$ is a conjugacy between $f$ and $g$.

Note that $d(p, q) \leqq d_{0}(\mathrm{id}, \varphi)$ where id denotes the identity map of $M$.

Suppose $f$ is strongly structurally stable and $\varepsilon>0$ is given. The physicist takes for $N_{0}$ the set of all $\varphi \in C^{0}(M, M)$ with $d_{0}$ (id, $\left.\varphi\right)<\varepsilon$; he finds that the neighborhood $N$ of $f$ in Definition 1.10 is large enough to allow him to conclude that $g \in N$. Thus he knows the true attractor $q$ to within error $\varepsilon$ as $d(p, q) \leqq d_{0}(\mathrm{id}, \varphi)<\varepsilon$.

Suppose the system $S$ is in state $y$ which the physicist measures obtaining a state $x$ with experimental error $\delta$ (so $d(x, y)<\delta$ ). He notes that $d(x, M \backslash U) \geqq \varepsilon+\delta$. Let $z=\varphi^{-1}(y)$. Then $d(x, z) \leqq d(x, y)+d(\varphi(z), z)$ $<\delta+\varepsilon$. Thus $z \in U$ so that $y=\varphi(z) \in \varphi(U)=V$. Thus $\lim _{n \rightarrow \infty} g^{n}(y)=q$. The physicist now knows that the system tends to an equilibrium state $q$ and he knows $q$ within error $\varepsilon$ (as $d(p, q)<\varepsilon$ ).

Next we consider an even stronger concept of structural stability: absolute structural stability. The definition is essentially due to Franks [9]; the improved version we give here is due to Guckenheimer [12]. It is motivated by mathematical considerations; a theorem due to Franks, Guckenheimer, and myself (see $\S 9$ ) gives a nice geometric characterization of it. This concept can also be given a nonmathematical motivation: If $f$ is absolutely structurally stable our physicist can easily estimate the error in his quantitative predictions (i.e. the size of $N_{0}$ ) in terms of his experimental error (i.e. the size of $N$ ). (See Definition 1.10.)

1.11. Definition. Let $f \in \operatorname{Diff}(M)$. A conjugacy selector for $f$ is a function (not necessarily continuous) $\Phi: N \rightarrow C^{0}(M, M)$ where $N$ is a neighborhood of $f$ in $\operatorname{Diff}(M)$ such that $\Phi(f)=$ identity and for all $g \in N, \Phi(g)$ is a homeomorphism and a conjugacy between $f$ and $g$.

Thus $f$ is structurally stable iff there exists a conjugacy selector for $f$; $f$ is strongly structurally stable iff there exists a conjugacy selector for $f$ which is continuous at $f$.

Now suppose $M$ is given a Riemannian metric. This determines a metric $d$ on $M$ which in turn determines a metric $d_{0}$ on $C^{0}(M, M)$ as above.

1.12. Definition. Let $f \in \operatorname{Diff}(M)$. Then $f$ is absolutely structurally stable iff there exists a conjugacy selector $\Phi: N \rightarrow C^{0}(M, M)$ for $f$ and a real number $K>0$ such that $d_{0}(\mathrm{id}, \Phi(g)) \leqq K d_{0}(f, g)$ for $g \in N$.

This definition is easily seen to be independent of the choice of the Riemannian metric in case $M$ is compact. It is a kind of Lipschitz condition, but note that the metric $d_{0}$ does not give the topology of $N$ (which is the $C^{1}$ topology). Thus the Lipschitz condition in 1.12 does not ob- 
viously follow from the mean value theorem and the condition that $\Phi: N \rightarrow C^{0}(M, M)$ be a $C^{1}$ map of Banach manifolds. ${ }^{4}$

1D. Criticism. Once we see the definition of structural stability and its motivation we are immediately tempted to change the definition. Thus, if $f$ is constructed via a physical theory, it will likely have special properties (e.g. preserve some structure) and if the theory is correct, the true system $g$ will share these properties. This suggests restricting $f$ and $g$ to some subset $\mathscr{F}$ of $\operatorname{Diff}(M)$. Also the topology on $\mathscr{F}$ which measures the experimental error might not be the topology on $\mathscr{F}$ induced by the $C^{1}$ topology on $\operatorname{Diff}(M)$. These ideas are easily incorporated into a definition.

1.13. Definition. Let $M$ be a smooth manifold and $\mathscr{F}$ be a subset of $\operatorname{Diff}(M)$ endowed with a topology possibly different from the induced topology. Then $f \in \mathscr{F}$ is structurally stable relative to $\mathscr{F}$ iff there exists a neighborhood $N$ of $f$ in $\mathscr{F}$ such that each $g \in N$ is topologically conjugate to $f$.

For many examples of $\mathscr{F}$, structural stability relative to $\mathscr{F}$ is equivalent to structural stability. For example, this is true when $\mathscr{F}$ is the set of gradient diffeomorphisms ${ }^{5}$ (with the $C^{1}$ topology) on a compact Riemannian manifold $M$. In $\S 2$ we show that it is also true when $M=\boldsymbol{R}^{m}$ and $\mathscr{F}=L_{\text {aut }}\left(\boldsymbol{R}^{m}\right)$, the set of all linear automorphisms of $\boldsymbol{R}^{m}$; here $\mathscr{F}$ has the topology it inherits as an open subset of the finite dimensional vector space $L\left(\boldsymbol{R}^{m}, \boldsymbol{R}^{m}\right)$ of linear operators on $\boldsymbol{R}^{m}$.

However, as C. Robinson pointed out to me, Definition 1.13 fails to cover many cases of interest in physics. Let $\mathscr{F}$ be the set of symplectic diffeomorphisms of a symplectic two-manifold $M$ (see [2]). Let $x \in M$ be an elliptic fixed point of $f \in \mathscr{F}$. According to the Moser twist stability theorem (see [2, p. 186]) there will be (generically, see [43]) a family of $f$-invariant, simple closed curves $\gamma$ converging to $x$ (see Figure 2). The rotation number (see [13]) of $f$ restricted to a typical $\gamma$ approaches a limit as $\gamma$ converges to $x$. This limit is a topological invariant which can be different for $g \in \mathscr{F}$ close to $f$. Thus $f$ is not structurally stable relative to $\mathscr{F}$. It seems that a new concept of structural stability is needed to cover this case. ${ }^{6}$

We next indicate the consequences of two obvious changes in the Definition 1.6 of structural stability. First, if arbitrary $C^{0}$ perturbations $g$ of $f$ are allowed, $f$ will never be structurally stable (in this new sense).

\footnotetext{
${ }^{4}$ Nonetheless, I conjecture that absolute structural stability is equivalent to structural stability.

${ }_{5}^{5} \mathrm{~A}$ gradient diffeomorphism is one of form $f^{1}$ where $f^{t}$ is the flow of a gradient vector field.

${ }^{6}$ [2] contains a good introduction to the theory of symplectic manifolds and their relation to classical mechanics. The introduction and conclusion of [2] also contain interesting philosophical remarks on stability questions.
} 
For if $x$ is an isolated fixed point of $f$ then there exists a $C^{0}$ perturbation

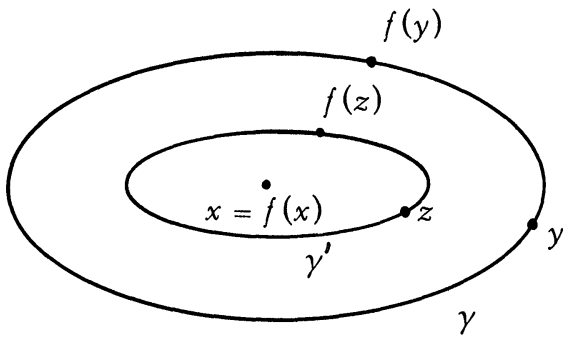

Figure 2. The Moser Twist Stability Theorem.

$g$ of $f$ having a tiny disc of fixed points about $x$ so $g$ and $f$ are not topologically conjugate. In this regard, the concept of "topological stability" (also called "semistability") of Walters [54] is of interest. This definition allows arbitrary $C^{0}$ perturbations but weakens the notion of conjugacy. Nitecki [30] is able to show that all known examples of structurally stable systems are semistable in this sense.

Secondly, if one modifies Definition 1.6 of structural stability by replacing the words "topologically conjugate" by the words "differentiably conjugate", one obtains a definition of structural stability which is never satisfied if $M$ is compact. The reason is as follows: From the $C^{1}$ genericity of weak axiom A (see $\S 7$ ) and the fact that the nonwandering set is always nonempty when $M$ is compact, it follows that there is a dense subset of $\operatorname{Diff}(M)$ each element of which has a periodic point and also has only finitely many periodic points of each period. Let $g=$ $\varphi \circ f \circ \varphi^{-1}$ where $g, \varphi$, and $f$ are all diffeomorphisms and suppose $x$ is a periodic point of $f$ (say $f^{n}(x)=x$ ) so that $y=\varphi(x)$ is a periodic point of $g$. Differentiating yields

$$
T_{y} g^{n}=\left(T_{x} \varphi\right)\left(T_{x} f^{n}\right)\left(T_{x} \varphi\right)^{-1},
$$

which shows that the linear operators $T_{y} g^{n}$ and $T_{x} f^{n}$ have the same eigenvalues. These eigenvalues can be changed by a small perturbation. It follows easily that no $f$ can be stable in this new sense.

Of course one might be able to demand differentiable conjugacies if at the same time one radically restricts the class of allowable perturbations as in Definition 1.13. Sternberg's linearization theorem (see [13]) is a theorem of this kind. One might restrict attention to $C^{r}$ diffeomorphisms $(r \geqq 2)$ without periodic points and allow only $C^{r}$ perturbations and expect stability with differentiable conjugacies. ${ }^{7}$ Alternatively, one might

\footnotetext{
${ }^{7}$ We assume here that the $C^{r}$ closing lemma is false. See [37] and [38].
} 
allow only perturbations supported in the complement of the closure of the periodic points. I know of no theorems in these directions.

Finally, I should point out that if one of these definitions is to be used by a physicist as envisioned above, then he will have to compute some, but not all, of the quantities asserted to exist. Thus to use 1.12 for example he will want to know $N$ and $K$ but not $\Phi(g)$ (since he does not know $g$ ). Thus partially constructive proofs of stability will be of more use to the physicist than nonconstructive proofs. Of course, the nonconstructive proofs are still important; they clarify the internal mathematical structure of the theory and provide encouragement to someone who actually wants to compute $N$ and $K$.

1E. The general problems. Two seem to be natural:

Problem I. Given a class $\mathscr{F}$ of discrete dynamical systems, characterize the structurally stable systems of $\mathscr{F}$.

Problem II. Given a class $\mathscr{F}$ of discrete dynamical systems, classify the elements of $\mathscr{F}$ up to topological conjugacy.

In Problem I, one may replace structural stability (1.6) by any other kind of stability (e.g., 1.10, 1.12 or 1.13).

These problems are vaguely stated and admit many different kinds of solutions. For example, there is always the trivial solution ( $f$ is stable if and only if $f$ is stable). Good solutions will involve conditions which are either easy to verify or theoretically interesting. The smaller the class $\mathscr{F}$, the more specific the conditions should be.

In $\$ 2$ and 3 we shall outline solutions to these problems for $\mathscr{F}=$ set of linear automorphisms of $\boldsymbol{R}^{m}$. The remainder of the paper is mostly devoted to Problem I (in the case of absolute structural stability) where $\mathscr{F}=\operatorname{Diff}(M)$ and $M$ is compact.

An ideal solution to Problem II would be for the case where $\mathscr{F}$ is a very large (i.e. residual) subset of $\operatorname{Diff}(M)$. This problem has been called the "Yin-Yang" problem by Abraham [1] because of its analogy to Oriental philosophy. It is probably too difficult to admit a reasonable solution.

2. Structural stability of linear automorphisms. In this section we outline a solution of Problem I in case $\mathscr{F}=L_{\text {aut }}\left(\boldsymbol{R}^{m}\right)$, the set of linear automorphisms of $\boldsymbol{R}^{m}$. The following definition is crucial.

2.1. Definition. Let $\boldsymbol{E}$ be a Banach space and $F$ a continuous linear automorphism of $\boldsymbol{E}$. Then $F$ is hyperbolic iff its spectrum contains no complex number of modulus one.

We shall also say that $F$ is a pure contraction when its spectrum lies 
entirely inside the unit circle (i.e. in the set $|z|<1$ ), that $F$ is a pure expansion when its spectrum lies entirely outside the unit circle (i.e. in the set $|z|>1$ ), and that $F$ is totally nonhyperbolic when its spectrum lies entirely on the unit circle $|z|=1$.

Now let $f \in L_{\text {aut }}\left(\boldsymbol{R}^{m}\right)$ and $\lambda \in C$. Let $E_{\lambda}(f) \subseteq \boldsymbol{R}^{m}$ be the largest vector subspace of $\boldsymbol{R}^{m}$, invariant by $f$, and which is such that the only eigenvalues of $f$ restricted to this subspace are $\lambda$ and $\bar{\lambda}$. When $\lambda$ is real this is the generalized eigenspace of $\lambda$; i.e. the kernel of $(f-\lambda)^{m}$. In general, $\boldsymbol{R}^{m}$ is the direct sum of the $E_{\lambda}(f)$ as $\lambda$ ranges over the eigenvalues of $f$.

Let

$$
\begin{aligned}
& W^{+}(f)=\sum_{|\lambda|<1} E_{\lambda}(f), \\
& W^{-}(f)=\sum_{|\lambda|>1} E_{\lambda}(f), \\
& W^{0}(f)=\sum_{|\lambda|=1} E_{\lambda}(f),
\end{aligned}
$$

and

$$
f_{e}=f \mid W^{e}(f)
$$

for $e=+,-, 0$. Thus by linear algebra we have an $f$-invariant direct sum decomposition

$$
\begin{aligned}
\boldsymbol{R}^{m} & =W^{+}(f) \oplus W^{-}(f) \oplus W^{0}(f), \\
f & =f_{+} \oplus f_{-} \oplus f_{0} .
\end{aligned}
$$

Note that $f_{+}$is a pure contraction, $f_{-}$is a pure expansion, and $f_{0}$ is totally nonhyperbolic. Moreover, $f$ is hyperbolic iff $W^{0}(f)=0$. The spaces $W^{ \pm}(f)$ admit a nice topological description.

2.2. LeMMA. I. $x \in W^{+}(f)$ if and only if $\lim _{n \rightarrow \infty} f^{n}(x)=0$.

II. $x \in W^{-}(f)$ if and only if $\lim _{n \rightarrow \infty} f^{-n}(x)=0$.

III. Given a norm on $\boldsymbol{R}^{m}$ there exist constants $c>0$ and $r$ with $0<r<1$ such that for $n \geqq 0$ :

$$
\left\|f^{n}(x)\right\| \leqq c r^{n}\|x\|, \quad \text { for } x \in W^{+}(f),
$$

and

$$
\left\|f^{-n}(x)\right\| \leqq c r^{-n}\|x\|, \quad \text { for } x \in W^{-}(f) .
$$

Moreover, the norm can be chosen so that $c=1$.

The proof of the lemma is an exercise in linear algebra which is left to the reader. The lemma motivates the following terminology: $W^{+}(f), W^{-}(f)$, and $W^{0}(f)$ are called respectively the in-set, the out-set, and the on-set of $f$. (See Figure 3.)

Problem I is now solved by 


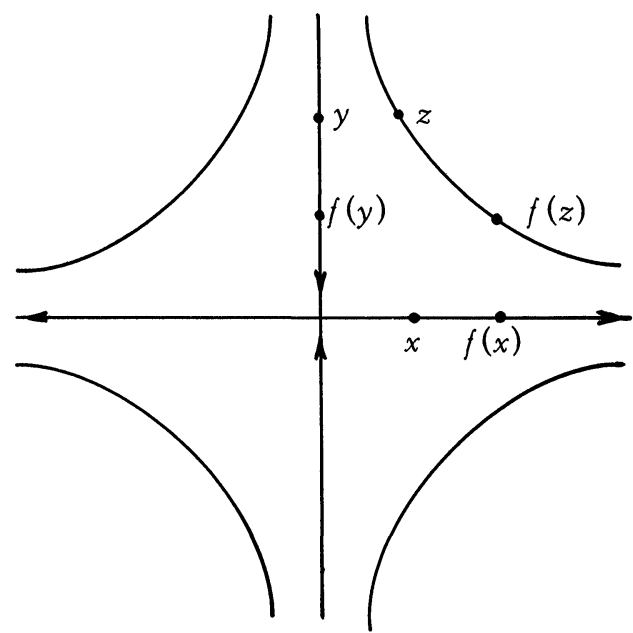

FigURE 3. $f\left(x_{1}, x_{2}\right)=\left(2 x_{1}, \frac{1}{2} x_{2}\right) . f$ is a hyperbolic linear automorphism of $\boldsymbol{R}^{2} ; W^{+}(f)=$ $0 \times \boldsymbol{R}, W^{-}(f)=\boldsymbol{R} \times 0$. Note that the hyperbolas $x_{1} x_{2}=$ constant are invariant under $f$.

2.3. HARTMAN's TheOREM. A linear automorphism $f$ of $\boldsymbol{R}^{m}$ is structurally stable if and only if it is hyperbolic.

Proof . "only if". Assume $f$ is not hyperbolic; i.e. $W^{0}(f) \neq 0$. Then there exist many points $x \in W^{0}(f)$ such that the orbit of $x$ by $f$ is bounded and $\lim _{n \rightarrow \infty} f^{n}(x) \neq 0$. Compose $f$ with a "contraction" which is $C^{1}$ close to the identity and this property is destroyed. Thus $f$ is not structurally stable.

"if". ${ }^{8}$ Given $\xi: \boldsymbol{R}^{m} \rightarrow \boldsymbol{R}^{m}$, define $\|\xi\|_{0}$ by

$$
\|\xi\|_{0}=\sup _{x \in R^{m}}\|\xi(x)\|
$$

and if $\xi$ is $C^{1}$ define $\|\xi\|_{1}$ by

$$
\|\xi\|_{1}=\|\xi\|_{0}+\sup _{x \in R^{m}}\|D \xi(x)\| .
$$

We denote by $B$ the Banach space of all continuous functions $\eta: R^{m} \rightarrow R^{m}$ with $\|\eta\|_{0}<\infty$. We let id denote the identity of $\boldsymbol{R}^{m}$ and 1 denote the identity of $B$.

Suppose $g$ is a $C^{1}$ approximation to $f$. Let $\xi=f^{-1} \circ g-$ id so that $g=f \circ(\mathrm{id}+\xi)$ and $\|\xi\|_{1}$ is small if $g$ is $C^{1}$ close to $f$. We seek a homeomorphism $\varphi: \boldsymbol{R}^{m} \rightarrow \boldsymbol{R}^{m}$ of the form $\varphi=\mathrm{id}+\eta$, where $\eta \in \boldsymbol{B}$ such that

\footnotetext{
${ }^{8}$ Hartman's original proof can be found in [13] or [14]. The proof we give here is due independently to Palis [32], Pugh [39], and myself and is inspired by Moser [25].
} 


$$
g \circ \varphi=\varphi \circ f
$$

Then (1) takes the form

$$
\left(1-f^{\#}\right) \eta=R_{\xi}(\eta)
$$

where

$$
f^{\#} \eta=f^{-1} \circ \eta \circ f
$$

and

$$
R_{\xi}(\eta)=-\xi \circ(\mathrm{id}+\eta) .
$$

Clearly $f^{\#}$ is a bounded linear operator on $\boldsymbol{B}$ and $R_{\xi}: B \rightarrow B$ has a small Lipschitz constant; in fact, by the mean value theorem,

$$
\left\|R_{\xi}\left(\eta_{1}\right)-R_{\xi}\left(\eta_{2}\right)\right\|_{0} \leqq\|\xi\|_{1}\left\|\eta_{1}-\eta_{2}\right\|_{0}
$$

for $\eta_{1}, \eta_{2} \in \boldsymbol{B}$.

Now $1-f^{\#}$ is invertible. To see this note that $\boldsymbol{B}=\boldsymbol{B}^{+} \oplus \boldsymbol{B}^{-}$, where $\boldsymbol{B}^{ \pm}$is the set of all $\eta \in \boldsymbol{B}$ such that $\eta: \boldsymbol{R}^{m} \rightarrow W^{ \pm}(f)$. (This is because $f$ is hyperbolic so $\boldsymbol{R}^{m}=W^{+}(f) \oplus W^{-}(f)$.) Thus

$$
\left(1-f^{\#}\right)^{-1} \zeta=\sum_{n=0}^{\infty} f^{\# n \zeta}
$$

for $\zeta \in \boldsymbol{B}^{-}$and

$$
\left(1-f^{\#}\right)^{-1} \zeta=-\sum_{n=1}^{\infty} f^{\#-n \zeta}
$$

for $\zeta \in \boldsymbol{B}^{+}$(Neumann series) and these series converge uniformly by Lemma 2.2, III.

Thus $\left(1^{\prime}\right)$ can be written

$$
\eta=\left(1-f^{\#}\right)^{-1} R_{\xi}(\eta) .
$$

By (3) the Lipschitz constant for the nonlinear operator on the right is bounded by $\left\|\left(1-f^{\#}\right)^{-1}\right\|\|\xi\|_{1}$ which is less than one if $\|\xi\|_{1}$ is small. Thus for $g$ close to $f$, (4) has a unique solution $\eta \in \boldsymbol{B}$ and thus (1) has a solution.

To complete the proof one must show that $\varphi$ is a homeomorphism. This is done by reversing the roles of $f$ and $g$ and using the uniqueness of the solution $\eta$. We refer the reader to [39] (for example) for details.

We next consider other notions of stability. Let $\mathscr{F}=L_{\text {aut }}\left(\boldsymbol{R}^{m}\right)$ have the topology it inherits as an open subset of $L\left(\boldsymbol{R}^{m}, \boldsymbol{R}^{m}\right)$, the finite dimensional vector space of all linear operators on $\boldsymbol{R}^{m}$. This topology is much weaker than the $C^{1}$ topology; if $f, g \in \mathscr{F}$ are distinct, then $\|f-g\|_{1}$ $\geqq\|f-g\|_{0}=\infty$. 
2.4. THEOREM. Let $f \in \mathscr{F}=L_{\text {aut }}\left(\boldsymbol{R}^{m}\right)$. Then the following are equivalent:

I. $f$ is hyperbolic;

II. $f$ is structurally stable;

III. $f$ is structurally stable relative to $\mathscr{F}$;

IV. $f$ is absolutely structurally stable.

Proof. We have already shown I if and only if II. The proof that III implies I is essentially the same as the proof that II implies I given above. Thus if $W^{0}(f) \neq 0$, then $f$ has bounded orbits other than 0 . Approximate $f$ by $g \in \mathscr{F}$ with $W^{0}(g)=0$. Then $g$ has no bounded orbit (other than 0 ) and is therefore not conjugate to $f$. This shows III implies I.

To prove I implies III we use Hartman's theorem and a trick. First we consider a special case: Suppose $f$ is a pure contraction. Let $\theta: \boldsymbol{R}^{m} \rightarrow \boldsymbol{R}$ be a smooth nonnegative function with compact support and equal to 1 on a neighborhood $U$ of 0 . Given $g \in \mathscr{F}$ consider $h: \boldsymbol{R}^{m} \rightarrow \boldsymbol{R}^{m}$ defined by

$$
h(x)=\theta(x) g(x)+(1-\theta(x)) f(x)
$$

for $x \in \boldsymbol{R}^{m}$. Then $h|U=g| U$ and $h$ tends to $f$ in $\operatorname{Diff}\left(\boldsymbol{R}^{m}\right)$ as $g$ tends to $f$ in $\mathscr{F}$. Thus for $g$ close to $f$ in $\mathscr{F}$, Hartman's theorem gives a homeomorphism $\psi: \boldsymbol{R}^{m} \rightarrow \boldsymbol{R}^{m}$ with $h \circ \psi=\psi \circ f$, whence $g \circ \psi(x)=\psi \circ f(x)$ for $x \in \psi^{-1}(U)$. Now define $\varphi: \boldsymbol{R}^{m} \rightarrow \boldsymbol{R}^{m}$ by

$$
\varphi(x)=g^{-n} \circ \psi \circ f^{n}(x)
$$

for $x \in \boldsymbol{R}^{m}$ where $n \geqq k$ and $f^{n}(x) \in \psi^{-1}(U)$ for all $n \geqq k$ (as $f$ is a pure contraction). Then $\varphi$ is well defined (the definition is independent of $n$ ) and clearly satisfies $g \circ \varphi=\varphi \circ f$. This proves the special case.

For the general case assume that $f$ is hyperbolic and $g$ is close to $f$ in $\mathscr{F}$. Then $g$ is also hyperbolic and the splittings $\boldsymbol{R}^{m}=W^{+}(f) \oplus W^{-}(f)$ and $\boldsymbol{R}^{m}=W^{+}(g) \oplus W^{-}(g)$ are close. By replacing $g$ with something conjugate to it by a linear conjugacy close to the identity we may assume that $W^{ \pm}(f)=W^{ \pm}(g)$. By the special case note that $f_{ \pm}$and $g_{ \pm}$are topologically conjugate. Taking the direct product gives that $f$ and $g$ are conjugate proving I implies III.

Clearly IV implies II. To prove I implies IV we examine the proof of Hartman's theorem given above. There we found $\eta=\varphi-$ id as the unique fixed point of the contraction map $\Gamma: \boldsymbol{B} \rightarrow \boldsymbol{B}$ given by (see (4))

$$
\Gamma(\eta)=-\left(1-f^{\#}\right)^{-1} \xi \circ(\mathrm{id}+\eta)
$$

for $\eta \in \boldsymbol{B}$. Note that

$$
\|\Gamma(0)\|_{0} \leqq c\|\xi\|_{0}
$$

for suitable $c$. Next recall 
2.5. Banach's Contraction Principle. Let $B$ be a closed ball about the origin in a Banach space (or possibly the whole Banach space) and let $\Gamma: B \rightarrow B$ satisfy

$$
\left\|\Gamma\left(\eta_{1}\right)-\Gamma\left(\eta_{2}\right)\right\| \leqq a\left\|\eta_{1}-\eta_{2}\right\|
$$

for $\eta_{1}, \eta_{2} \in B$ (where $0<a<1$ ) and $\|\Gamma(0)\| \leqq \varepsilon$. Then there exists a unique $\eta \in B$ such that $\Gamma(\eta)=\eta$. Moreover, $\|\eta\| \leqq \varepsilon /(1-a)$.

To prove 2.5 let $\eta_{n}=\Gamma^{n}(0)$. As

$$
\sum_{n=0}^{\infty}\left\|\eta_{n+1}-\eta_{n}\right\| \leqq \sum_{n=0}^{\infty} a^{n}\left\|\eta_{1}\right\| \leqq \frac{\varepsilon}{1-a},
$$

it follows that $\lim \eta_{n}=\eta$ exists and satisfies the conclusions of 2.5. This is of course the standard proof.

Now

$$
d_{0}(\varphi, \mathrm{id})=\|\varphi-\mathrm{id}\|_{0}=\|\eta\|_{0}
$$

and

$$
d_{0}(g, f)=\|g-f\|_{0}=\|f \circ \xi\|_{0} \geqq b\|\xi\|_{0}
$$

and as we may take $a=\frac{1}{2}$ for $\|\xi\|_{1}$ small we obtain

$$
d_{0}(\varphi, \mathrm{id}) \leqq K d_{0}(g, f)
$$

for suitable $K$, proving I implies IV.

3. Classification of linear automorphisms. We now turn to Problem II of $\S 1 \mathrm{E}$ for the case $\mathscr{F}=L_{\text {aut }}\left(\boldsymbol{R}^{m}\right)$. We retain the notation of $\S 2$. Let $\operatorname{Hyp}\left(\boldsymbol{R}^{m}\right)$ denote the set of all hyperbolic linear automorphisms of $\boldsymbol{R}^{\boldsymbol{m}}$; note that $\operatorname{Hyp}\left(\boldsymbol{R}^{m}\right)$ is open dense in $L_{\text {aut }}\left(\boldsymbol{R}^{m}\right)$. For $f \in L_{\text {aut }}\left(\boldsymbol{R}^{m}\right)$ let

$$
\begin{array}{ll}
w^{ \pm}(f)=\operatorname{dim} W^{ \pm}(f), & \text { if } f \mid W^{ \pm}(f) \text { preserves orientation; } \\
w^{ \pm}(f)=-\operatorname{dim} W^{ \pm}(f) & \text { otherwise. }
\end{array}
$$

3.1. TheOREM . For $f, g \in \operatorname{Hyp}\left(\boldsymbol{R}^{m}\right)$ the following are equivalent:

I. $f$ and $g$ are topologically conjugate;

II. $w^{+}(f)=w^{+}(g)$ and $w^{-}(f)=w^{-}(g)$;

III. $f$ and $g$ belong to the same component of $\operatorname{Hyp}\left(\boldsymbol{R}^{m}\right)$.

Proof. For I implies II note that the numbers $w^{ \pm}(f)$ are topological invariants by Lemma 2.2. II implies III is an exercise in linear algebra which we leave to the reader. (Hint: use Jordan normal form.) For III implies I connect $f$ and $g$ by a curve, use 2.4 (I implies III), and apply "continuous induction." Note that a corollary of 3.1 is that $\operatorname{Hyp}\left(\boldsymbol{R}^{m}\right)$ has $4 m$ components. (Recall Example 1.5.)

We now attempt to generalize 3.1 : 
3.2. Demitheorem . ${ }^{9}$ Let $f, g \in L_{\text {aut }}\left(\boldsymbol{R}^{m}\right)$. Then $f$ and $g$ are topologically conjugate if and only if $w^{+}(f)=w^{+}(g), w^{-}(f)=w^{-}(g)$, and $f_{0}$ and $g_{0}$ are linearly conjugate.

Now 3.2 clearly implies:

3.3. Demicorollary. Totally nonhyperbolic linear automorphisms are topologically conjugate if and only if they are linearly conjugate.

Note 3.3 has as a special case:

3.4. Conjecture. Periodic linear automorphisms are topologically conjugate if and only if they are linearly conjugate.

Kuiper and I [21] have succeeded in proving 3.2 from 3.4 and we can also prove 3.2 under the additional assumption that $f$ and $g$ have no periodic points of prime period $>3$.

Unfortunately, 3.4 appears to be very difficult. de Rham ([40] and [41]) proves a weaker version of 3.4. Note that 3.4 implies the topological classification of lens spaces, a problem which was open for at least thirty years and solved only recently by the deep work of Kirby and Siebenmann [20].

We close this section by proving two special cases of the demicorollary; the proofs indicate the main ideas in the general case.

3.5. EXAMPLE. Irrational (i.e. nonperiodic) rotations of $\boldsymbol{R}^{2}$ are topologically conjugate if and only if they are linearly conjugate.

Proof. Let $f$ rotate $\boldsymbol{R}^{2}=\boldsymbol{C}$ through an irrational angle;

$$
f(z)=2^{\pi i \theta} z
$$

for $z \in C$ where $\theta$ is irrational. For $z \in C, z \neq 0$, let $S$ be the closure of the orbit of $z$. Then $S$ is a circle and is invariant under $f$. Moreover, the rotation number (see [13]) of $f \mid S$ is $\theta$. As this number is a topological invariant which characterizes $f$ up to linear conjugacy, 3.5 is proved.

Now let $h_{k} \in L_{\text {aut }}\left(\boldsymbol{R}^{k}\right)$ be the automorphism represented by the matrix with ones on the diagonal and super diagonal and zeros elsewhere. Define $f, g \in L_{\text {aut }}\left(\boldsymbol{R}^{4}\right)$ by $f=h_{2} \times h_{2}$ and $g=h_{3} \times h_{1}$. Then $f$ and $g$ are totally nonhyperbolic ( 1 is the only eigenvalue) and not linearly conjugate as they have different Jordan normal forms.

3.6. EXAMPLE $f$ and $g$ are not topologically conjugate.

Proof. Let $X(f) \subseteq \boldsymbol{R}^{4}$ be the set of nonfixed points of $f$. Then $f$ acts freely on $X(f)$ and the orbit space $X(f) / f$ is a topological invariant associated to $f$. We prove 3.6 by showing that $X(f) / f$ is Hausdorff while $X(g) / g$ is not.

\footnotetext{
${ }^{9} \mathrm{~A}$ demitheorem is an assertion which is almost completely proved and for which there is overwhelming evidence of its truth.
} 
To do this we examine the orbit structure of $h_{k}(k=2,3)$. The $h_{2}$ orbit of a point $(x, y) \in \boldsymbol{R}^{2}$ lies on a line $y=$ constant. This point is fixed if and only if $y=0$. Thus $X\left(h_{2}\right) / h_{2}$ is a disjoint union of two open cylinders.

The $h_{3}$ orbit of a point $(x, y, z) \in \boldsymbol{R}^{3}$ lies on a parabola if $z \neq 0$; lies on a straight line $y=$ constant and $z=0$ if $z=0$; and is fixed (i.e. consists of a single point) if $y=z=0$. The parabola for $z \neq 0$ approaches two distinct lines $(y= \pm$ constant, $z=0)$ as $z$ tends to 0 with $y \neq 0$. This is why $X\left(h_{3}\right) / h_{3}$ is not Hausdorff. Using these ideas it is not hard to prove 3.6.

4. Structural stability on a compact manifold. We now consider Problem I of $\S 1 \mathrm{E}$ in case $\mathscr{F}=\operatorname{Diff}(M)$ where $M$ is compact. We first introduce some notation which will remain in force for the remainder of the paper.

For each $r=0,1,2, \ldots, C^{r}(T M)$ will denote the Banachable space of $C^{r}$ vector fields on $M$. We fix, once and for all, a norm $\|\cdot\|_{r}$ on this space.

We shall take a smooth exponental map exp: $T M \rightarrow M$ for $M$. It may be constructed by taking a smooth Riemannian metric on $M$ and setting $\exp (\dot{x})=c(1)$ for $\dot{x} \in T M$ where $c: R \rightarrow M$ is the unique geodesic satisfying $\dot{c}(0)=\dot{x}$. For us, the most important fact about exp is the following: Let $U$ be a coordinate patch on $M$ (so we identify $U$ with an open subset of $\left.\boldsymbol{R}^{m}\right)$. Then for $\dot{x}=(x, v) \in U \times \boldsymbol{R}^{m}=T U$,

$$
\exp (\dot{x})=x+v+\ldots
$$

where the dots represent terms of higher order.

Given $\eta \in C^{0}(T M)$ we define $\exp (\eta): M \rightarrow M$ by $\exp (\eta)=\exp \circ \eta$. Thus according to (1), $\exp (\eta)$ is the analog of id $+\eta$ in $\S 2$. Using (1) and the implicit function theorem it is not hard to show for a sufficiently small ball $B$ about the origin in $C^{0}(T M)$ that the map

$$
B \cap C^{r}(T M) \rightarrow C^{r}(M, M): \eta \rightarrow \exp (\eta)
$$

is a homeomorphism onto a neighborhood of the identity in $C^{r}(M, M)$. (Indeed, it is a chart on the Banach manifold $C^{r}(M, M)$; see [31].)

Now suppose we want to show that $f \in \operatorname{Diff}(M)$ is structurally stable. We choose $g$ close to $f$ in $\operatorname{Diff}(M)$ and try to solve for $\varphi$ in the equation

$$
g \circ \varphi=\varphi \circ f .
$$

Following Moser [25] we note that $f^{-1} \circ g$ is close to the identity so $f^{-1} \circ g=\exp (\xi)$ where $\xi \in C^{1}(T M)$ and $\|\xi\|_{1}$ is small. We seek a solution $\varphi$ near the identity $\varphi=\exp (\eta)$, where $\eta \in B \subseteq C^{0}(T M)$. Then (2) takes the form

$$
\left(1-f^{*}\right) \eta=R_{\xi}(\eta)
$$

where $f^{*}$ is the linear operator on $C^{0}(T M)$ defined by 


$$
f^{*} \eta=T f^{-1} \circ \eta \circ f
$$

for $\eta \in C^{0}(T M)$ and $R_{\xi}: B \rightarrow C^{0}(T M)$ has a small Lipschitz constant for $\|\xi\|_{1}$ small.

The reader should compare equations (2) and (2') with equations (1) and $\left(1^{\prime}\right)$ of $\S 2$.

4.1. Definition. Let $f \in \operatorname{Diff}(M)$. Then $f$ is an Anosov diffenmorphism iff the bounded linear operator $f^{\#}: C^{0}(T M) \rightarrow C^{0}(T M)$ is hyperbolic.

4.2. TheORem (ANosov [5]). An Anosov diffeomorphism $f$ is structurally stable.

Proof (Moser [25]). As $f^{*}$ is hyperbolic, 1 is not in its spectrum; i.e. $1-f^{\#}$ is invertible. Hence $\left(2^{\prime}\right)$ takes the form

$$
\eta=\left(1-f^{\#}\right)^{-1} R_{\xi}(\eta),
$$

and one can proceed as in the proof of 2.3 .

Unfortunately, this proof works only when $f$ is Anosov:

4.3. Theorem (MATHER [24]). Let $f \in \operatorname{Diff}(M)$. Then $f$ is Anosov if and only if $1-f^{*}$ is invertible.

Anosov diffeomorphisms are relatively rare; many manifolds do not even admit one. However, to find solutions of $\left(2^{\prime}\right)$, one does not need to invert $1-f^{*}$; one only needs a continuous linear right inverse $J: C^{0}(T M)$ $\rightarrow C^{0}(T M)$ to $1-f^{*}$. For if $\left(1-f^{*}\right) J=1$, then any solution of

$$
\eta=J R_{\xi}(\eta)
$$

is also a solution of $\left(2^{\prime}\right)$ (but not conversely). By the open mapping theorem, a continuous linear operator has a right inverse if and only if it is split surjective; i.e. it is surjective and its kernel has a closed complement. This leads us to the following

4.4. ConjeCture. ${ }^{10}$ Let $f \in \operatorname{Diff}(M)$. Then $f$ is structurally stable if and and only if $1-f^{*}$ is split surjective.

We shall now try to prove this conjecture.

4.5. Definition. Let $f \in \operatorname{Diff}(M)$. A selector for $f$ is a function $\Phi: N$ $\rightarrow C^{0}(M, M)$ where $N$ is a neighborhood of $f$ in (the Banach manifold) $\operatorname{Diff}(M)$ such that $\Phi(f)=$ id and for $g \in N$ we have $g \circ \varphi=\varphi \circ f$ where $\varphi=\Phi(g)$. The selector is absolute iff

$$
d_{0}(\mathrm{id}, \Phi(g)) \leqq K d_{0}(f, g)
$$

\footnotetext{
${ }^{10}$ I also conjecture that $f$ is structurally stable if and only if it is absolutely structurally stable and that $1-f^{\#}$ is split surjective if and only if it is surjective.
} 
for $g \in N$, where $K$ is a positive constant and $d_{0}$ is the $C^{0}$ metric of $\S 1 C$. The selector is differentiable iff it is differentiable as a map of Banach manifolds.

Note that a selector differs from a conjugacy selector (1.11) only in that $\Phi(g)$ need not be a homeomorphism.

4.6. THEOREM . Let $f \in \operatorname{Diff}(M)$ where $M$ is compact. Then

I. (Guckenheimer [12]). If $f$ admits an absolute selector, then $1-f^{*}$ is surjective.

II. (Franks [9]). $f$ admits a differentiable, absolute selector if and only if $1-f^{*}$ is split surjective.

Proof. Suppose $\Phi$ is an absolute selector for $f$. For $\xi \in C^{1}(T M)$ with $\|\xi\|_{1}$ small we may define $H(\xi) \in C^{0}(T M)$ by

$$
\Phi(f \circ \exp (\xi))=\exp (H(\xi)) .
$$

The absoluteness condition becomes

$$
\|H(\xi)\|_{0} \leqq k\|\xi\|_{0}
$$

for suitable positive constant $k$. Equation $\left(2^{\prime}\right)$ becomes

$$
\left(1-f^{\#}\right) H(\xi)=R_{\xi}(H(\xi)) \text {. }
$$

Choose $\xi \in C^{1}(T M)$ and a small positive real number $t$. In the last equation substitute $t \xi$ for $\xi$ and divide by $t$ :

$$
\left(1-f^{*}\right) t^{-1} H(t \xi)=t^{-1} R_{t \xi}(H(t \xi)) \text {. }
$$

A careful analysis of $R_{\xi}$ (see [12], [25], [45] and compare with equation (2) of \$2) shows that the right-hand side of (6) tends to $-\xi$ as $t$ tends to 0 . By (5),

$$
\left\|t^{-1} H(t \xi)\right\|_{0} \leqq k\|\xi\|_{0} .
$$

As $C^{1}(T M)$ is dense in $C^{0}(T M)$ this shows that the image under $1-f^{*}$ of the ball of radius $k$ in $C^{0}(T M)$ is dense in the unit ball of $C^{0}(T M)$. By a suitable formulation of the open mapping principle (see [23]) it follows that $1-f^{*}$ is surjective proving $\mathrm{I}$.

For the "only if" direction of II note that $H$ is differentiable if $\Phi$ is. Thus for $\xi \in C^{1}(T M), J \xi=-\lim _{t \rightarrow 0} t^{-1} H(t \xi)$ exists, and by (6) it satisfies $\left(1-f^{*}\right) J \xi=\xi$. By $(7),\|J \xi\|_{0} \leqq k\|\xi\|_{0}$ so that $J$ extends uniquely to $C^{0}(T M)$. We have constructed a right inverse $J$ to $1-f^{*}$ as required.

For the "if" direction of II let $J$ be a right inverse to $1-f^{\#}$. For $\xi \in C^{1}(T M)$ with $\|\xi\|_{1}$ small let $\eta=H(\xi) \in C^{0}(T M)$ be the unique solution of (3) (by the Banach contraction principle 2.5). As in the proof of 2.4 we have $\left\|J R_{\xi}(0)\right\|_{0} \leqq c\|\xi\|_{0}$ so that (5) holds. Now $\eta=H(\xi)$ is the unique 
solution of $G(\xi, \eta)=0$ where $G(\xi, \eta)=\eta-J R_{\xi}(\eta)$ is a $C^{1}$ function of $(\xi, \eta)$ defined on a neighborhood of 0 of the Banach space $C^{1}(T M)$ $\times C^{0}(T M)$ and with values in $C^{0}(T M)$. As $G(0,0)=0$ and $D_{2} G(0,0)=1$, the equation $G(\xi, \eta)=0$ may be solved for $\eta=H(\xi)$ by the implicit function theorem. Hence $H(\xi)$ is a $C^{1}$ function of $\xi$. It now follows that $\Phi$ defined by (4) is a differentiable, absolute selector for $f$. This completes our proof. For more details see [9] and [12].

The last argument fails to prove structural stability because the selector $\Phi$ need not be a conjugacy selector. Now the $\Phi$ constructed above depends on the choice of the right inverse $J$. Example 1.8 shows that $\Phi$ will not be a conjugacy selector if $J$ is badly chosen. Does a good choice of $J$ exist?

4.7. Definition [45]. Let $f \in \operatorname{Diff}(M)$. Then $f$ is infinitesimally stable iff there exists a right inverse $J$ to $1-f^{*}$ such that ... .

The dots represent a technical condition which assures that the selector $\Phi$ constructed from $J$ is in fact a conjugacy selector. Happily this condition is often satisfied.

4.8. TheOREM. Let $f \in \operatorname{Diff}(M)$ where $M$ is compact and suppose $f$ is $C^{2}$. Then $f$ is absolutely structurally stable if and only if $f$ is infinitesimally stable.

The reader should compare 4.8 and 4.4. We shall elaborate on 4.8 in $\S 9$ (Theorem 9.2).

5. The adjoint representation. Let $G$ be a Lie group and $\mathscr{G}$ be its Lie algebra. One can think of $\mathscr{G}$ as the tangent space to $G$ at the identity. Thus if $h_{t} \in G(t \in R)$ is a curve with $h_{0}=$ identity, then the tangent vector $\eta$ to this curve at time $t=0$ is an element of $\mathscr{G}$ and every element of $\mathscr{G}$ may be so obtained.

Let $f \in G$. Then $f$ determines an inner automorphism $\operatorname{Ad}(f): G \rightarrow G$ by $\operatorname{Ad}(f)(h)=f h f^{-1}$ for $h \in G$. Denote by $\operatorname{ad}(f): \mathscr{G} \rightarrow \mathscr{G}$ the tangent of $\operatorname{Ad}(f)$ at the identity. Thus if $\eta$ is the tangent vector to $h_{t}$ at $t=0$, then $\operatorname{ad}(f) \eta$ is the tangent vector to $f h_{t} f^{-1}$ at $t=0$. We thus obtain a representation ad: $G \rightarrow L_{\text {aut }}(\mathscr{G})$ of $G$ on $\mathscr{G}$. This is called the adjoint representation of $G$ and is the fundamental representation of Lie group theory.

Now let $M$ be a compact manifold. Its diffeomorphism group $\operatorname{Diff}(M)$ has many of the properties of a Lie group: It is a group and a Banach manifold. It is not an infinite dimensional Lie group because the group operations are not differentiable (but they are continuous).

To find the Lie algebra of $\operatorname{Diff}(M)$ choose a curve $h_{t} \in \operatorname{Diff}(M)$ with $h_{0}=$ identity and differentiate at $t=0$ : 


$$
\eta(x)=\left.(d / d t) h_{t}(x)\right|_{t=0}
$$

for $x \in M$. Clearly $\eta(x) \in T_{x} M$ for $x \in M$ which means that $\eta$ is a vector field on $M$. If $h_{t}$ is the flow of $\eta \in C^{1}(T M)$ then (1) holds. This suggests that the Lie algebra of $\operatorname{Diff}(M)$ should be $C^{1}(T M)$. (If we replace $C^{1}$ by $C^{\infty}$ we actually get a Lie algebra closed under Lie brackets.)

An $f \in \operatorname{Diff}(M)$ determines an inner automorphism $\operatorname{Ad}(f)$ by $\operatorname{Ad}(f) h$ $=f \circ h \circ f^{-1}$ for $h \in \operatorname{Diff}(M)$. If $h_{t}$ satisfies (1) then

$$
\left.(d / d t) \operatorname{Ad}(f) h_{t}\right|_{t=0}=T f \circ \eta \circ f^{-1} .
$$

The right-hand side makes sense for any vector field $\eta, C^{1}$ or not. Thus we make the following

5.1. Definition. ${ }^{11}$ Let $f \in \operatorname{Diff}(M)$ where $M$ is a compact smooth manifold. The adjoint representation of $f$ is the linear operator $\operatorname{ad}(f): C^{0}(T M)$ $\rightarrow C^{0}(T M)$ given by

$$
\operatorname{ad}(f) \eta=T f \circ \eta \circ f^{-1}
$$

for $\eta \in C^{0}(T M)$.

One easily verifies that $\operatorname{ad}(f)$ is a representation: $\operatorname{ad}(f \circ g)=\operatorname{ad}(f) \operatorname{ad}(g)$, $\operatorname{ad}(\mathrm{id})=1$, and $\operatorname{ad}\left(f^{-1}\right)=\operatorname{ad}(f)^{-1}$ for $f, g \in \operatorname{Diff}(M)$. In fact, if $f, \xi, \eta$ are sufficiently smooth we also have $\operatorname{ad}(f)[\xi, \eta]=[\operatorname{ad}(f) \xi, \operatorname{ad}(f) \eta]$.

Note that for $f \in \operatorname{Diff}(M), \operatorname{ad}\left(f^{-1}\right)=\operatorname{ad}(f)^{-1}=f^{\#}$, where $f^{\#}$ is the operator defined in $\S 4$. Thus the fundamental representation in the theory of dynamical systems is the analog of the fundamental representation of Lie group theory.

I cannot resist a brief digression to illustrate an application of adjoint representation to ergodic theory. Let $M$ be a compact smooth manifold, $\omega$ a smooth volume on $M$, and suppose $f \in \operatorname{Diff}(M)$ preserves $\omega: f^{*} \omega=\omega$. We denote by $H^{r}(T M)$ the Sobolev space of vector fields having $r$ derivatives in $L^{2}$. Similarly let $H^{r}\left(T^{*} M\right)$ denote the Sobolev space of $H^{r}$ one-forms on $M$ and $H^{r}(M)$ denote the Sobolev space of $H^{r}$ real-valued functions on $M$. Note that the exterior derivative $d$ maps $H^{r}(M)$ to $H^{r-1}\left(T^{*} M\right)$ and that $\operatorname{ad}(f)$ is a bounded linear operator on $H^{r}(T M)$ if $f$ is $C^{r+1}$.

5.2. Definition. $f$ is ergodic iff for all $q \in H^{0}(M)$ the equation $f^{*} q=q$ implies $q$ is constant.

5.3. Definition. $f$ is infinitesimally ergodic iff the operator $1-\operatorname{ad}(f)$ : $H^{1}(T M) \rightarrow H^{1}(T M)$ has dense range.

5.4. THEOREM. If $f$ is infinitesimally ergodic, then $f$ is ergodic.

11 This definition was told to me by Franks. 
Proof. Given a vector field $\eta$ and a one-form $\alpha$ define a real number $\langle\alpha, \eta\rangle$ by

$$
\langle\alpha, \eta\rangle=\int_{x \in M} \alpha(x) \eta(x) \omega(x) .
$$

This pairing determines the dual of $H^{r}(T M): H^{r}(T M)^{*}=H^{-r}\left(T^{*} M\right)$. As $f^{*} \omega=\omega$ the change of variables formula shows that $\langle\alpha, \operatorname{ad}(f) \eta\rangle$ $=\left\langle f^{*} \alpha, \eta\right\rangle$, where $f^{*}$ is the induced operator on forms. Suppose $f^{*} q=q$. Then $d q=d f^{*} q=f^{*} d q$ so

$$
(1-\operatorname{ad}(f))^{*} d q=\left(1-f^{*}\right) d q=0 .
$$

As $1-\operatorname{ad}(f)$ has dense range, $(1-\operatorname{ad}(f))^{*}$ has no kernel. Thus $d q=0$ so $q$ is constant. This proves 5.4.

We recall that (see 4.1) $f$ is Anosov if and only if $\operatorname{ad}(f)$ is hyperbolic on $C^{0}(T M)$; i.e. $\lambda-\operatorname{ad}(f): C^{0}(T M) \rightarrow C^{0}(T M)$ is an isomorphism for all $\lambda \in C$ with $|\lambda|=1$. The spaces $H^{1}(T M)$ and $C^{0}(T M)$ do not seem to be too different. Thus we have

5.5. Conjecture. If $f$ is Anosov, then $f$ is infinitesimally ergodic.

If true, the conjecture would imply a difficult theorem of Anosov: $C^{2}$ Anosov diffeomorphisms are ergodic (see [5]).

The conjecture is necessarily very delicate as Moser pointed out to me. For, let $f$ be a hyperbolic toral diffeomorphism (Example 1.9 of $\$ 1$ ). Then using Fourier series one can show that $1-\operatorname{ad}(f): H^{r}(T M) \rightarrow H^{r}(T M)$ is an isomorphism for $r<1$, has dense range for $r=1$, and does not have dense range for $r>1$. ( $r$ is here a real number.)

6. Hyperbolic sets. Let $f \in \operatorname{Diff}(M)$ and $\Lambda \subseteq M$. Let $T M \mid \Lambda \rightarrow \Lambda$ denote the restriction of the tangent bundle $T M \rightarrow M$ to $\Lambda$ and let $C^{0}(T M \mid \Lambda)$ denote the space of continuous sections of this restricted bundle. When $\Lambda$ is compact, $C^{0}(T M \mid \Lambda)$ is a Banachable space and the restriction map $C^{0}(T M) \rightarrow C^{0}(T M \mid \Lambda)$ is surjective (section extension theorem). When $\Lambda$ is $f$-invariant (i.e. $f(\Lambda)=\Lambda$ ) the adjoint operator $\operatorname{ad}(f): C^{0}(T M) \rightarrow C^{0}(T M)$ has a natural restriction $\operatorname{ad}(f, \Lambda): C^{0}(T M \mid \Lambda)$ $\rightarrow C^{0}(T M \mid \Lambda)$ given by

$$
\operatorname{ad}(f, \Lambda) \eta=T f \circ \eta \circ f^{-1}
$$

for $\eta \in C^{0}(T M \mid \Lambda)$.

6.1. Definition. Let $f \in \operatorname{Diff}(M)$. A compact, $f$-invariant subset $\Lambda \subseteq M$ is called hyperbolic (for $f$ ) iff the continuous linear operator $\operatorname{ad}(f, \Lambda)$ : $C^{0}(T M \mid \Lambda) \rightarrow C^{0}(T M \mid \Lambda)$ is hyperbolic.

Thus $\operatorname{ad}(f)=\operatorname{ad}(f, M)$. As $f^{*}=\operatorname{ad}(f)^{-1}$ is hyperbolic if and only if $\operatorname{ad}(f)$ is hyperbolic we see that $f$ is an Anosov diffeomorphism (see 4.1) if and only if $M$ is a hyperbolic invariant set of $f$. 
The following remarkable observation is due to Mather [24]; Theorem 4.3 is an easy corollary of it.

6.2. THEOREM. Let $f \in \operatorname{Diff}(M)$ and $\Lambda \subseteq M$ be compact and $f$-invariant. Suppose the nonperiodic points of $f$ in $\Lambda$ are dense in $\Lambda$. Then the spectrum of $\operatorname{ad}(f, \Lambda)$ is invariant under rotations of the complex plane. In particular, $\Lambda$ is hyperbolic if and only if $1-\operatorname{ad}(f, \Lambda)$ is bijective.

We now give an older, geometric definition of "hyperbolic invariant set"; the equivalence of these two definitions is due to Mather [24].

Let $X$ be a compact Hausdorff space and $E \rightarrow X$ a vector bundle over $X$. Let $C^{0}(X)$ denote the ring of continuous, real-valued functions on $X$ and let $C^{0}(E)$ denote the Banachable space of sections of $E \rightarrow X$. Thus $C^{0}(E)$ is a $C^{0}(X)$-module.

6.3. LEMMA. The correspondence which sends a vector bundle splitting $E=E^{+} \oplus E^{-}$to the corresponding splitting $C^{0}(E)=C^{0}\left(E^{+}\right) \oplus C^{0}\left(E^{-}\right)$of $C^{0}(X)$-modules is bijective.

This lemma is essentially the content of Swan's theorem [53] to the effect that topological $K$-theory is a special case of algebraic $K$-theory. To prove it one notes that splittings correspond to projections and that the $C^{0}(X)$-algebras $C^{0}(\operatorname{Hom}(E, E))$ and $\operatorname{Hom}\left(C^{0}(E), C^{0}(E)\right)$ are naturally isomorphic (see for example p. 26 of [19]).

Now let $\boldsymbol{E}$ be a Banach space and $\boldsymbol{F}$ a continuous linear automorphism of $\boldsymbol{E}$. Then $\boldsymbol{F}$ is hyperbolic if and only if there exists a closed, $F$-invariant splitting $\boldsymbol{E}=\boldsymbol{E}^{+} \oplus \boldsymbol{E}^{-}$of $\boldsymbol{E}$ and constants $c>0$ and $0<r<1$ such that for $n \geqq 0$

$$
\left\|F^{n} \eta\right\| \leqq c r^{n}\|\eta\| \quad \text { for } \eta \in E^{+}
$$

and

$$
\left\|F^{-n} \eta\right\| \leqq c r^{n}\|\eta\| \quad \text { for } \eta \in E^{-} .
$$

This fact is a generalization of Lemma 2.2 and is proved in any textbook on linear functional analysis.

Now take $\boldsymbol{E}=C^{0}(T M \mid \Lambda)$ and $F=\operatorname{ad}(f, \Lambda)$ where $\Lambda$ is compact and $f$-invariant. Suppose $M$ has a Riemannian metric so that a norm $\|\cdot\|_{0}$ on $C^{0}(T M \mid \Lambda)$ is determined by $\|\eta\|_{0}=\sup _{x \in \Lambda}\|\eta(x)\|$ for $\eta \in C^{0}(T M \mid \Lambda)$. If $\Lambda$ is hyperbolic, we see from $(1 \pm)$ that the subspaces $\boldsymbol{E}^{+}$and $\boldsymbol{E}^{-}$are $C^{0}(X)$-modules. Hence by 6.3 the splitting $C^{0}(T M \mid \Lambda)=E^{+} \oplus E^{-}$comes from a vector bundle splitting $T M \mid \Lambda=E^{+} \oplus E^{-}$where $E^{ \pm}=C^{0}\left(E^{ \pm}\right)$. This proves the following

6.4. Theorem. A compact, $f$-invariant set $\Lambda$ is hyperbolic if and only if there exists an f-invariant splitting of vector bundles $T M \mid \Lambda=E^{+} \oplus E^{-}$ 
and constants $c>0$ and $0<r<1$ such that for $n \geqq 0$

$$
\left\|T f^{n} \dot{x}\right\| \leqq c r^{n}\|\dot{x}\| \quad \text { for } \dot{x} \in E^{+}
$$

and

$$
\left\|T f^{-n} \dot{x}\right\| \leqq c r^{n}\|\dot{x}\| \quad \text { for } \dot{x} \in E^{-} .
$$

From Theorem 6.4 we easily see that a compact, $f$-invariant subset of a hyperbolic set is hyperbolic.

A very important example of a compact, $f$-invariant set is a periodic orbit

$$
\Lambda=\left\{x, f(x), f^{2}(x), \ldots, f^{n-1}(x)\right\}
$$

where $f^{n}(x)=x$. The reader can easily show that this $\Lambda$ is hyperbolic if and only if the linear automorphism $T_{x} f^{n}: T_{x} M \rightarrow T_{x} M$ is hyperbolic. Moreover, if $n$ is the prime period (i.e. $f^{k}(x) \neq x$ for $0<k<n$ ), then the spectrum of $\operatorname{ad}(f, \Lambda)$ is the set of all complex numbers $\lambda$ such that $\lambda^{n}$ is an eigenvalue of $T_{x} f^{n}$.

7. Axiom A. Let $f \in \operatorname{Diff}(M)$. The nonwandering set of $f$ is denoted by $\Omega(f)$ and is defined to be the set of all $x \in M$ such that for every neighborhood $U$ of $x$ there exists an integer $n \neq 0$ such that $f^{n}(U) \cap U=\varnothing$.

The set of periodic points of $f$ is denoted by $P(f)$ (so that $x \in P(f)$ iff there exists $n \neq 0$ with $\left.f^{n}(x)=x\right)$ and $\bar{P}(f)$ shall denote the closure of $P(f)$. Note that $\Omega(f)$ and $\bar{P}(f)$ are closed and $f$-invariant and that $\bar{P}(f) \subseteq \Omega(f)$. If $M$ is compact, $\Omega(f) \neq \varnothing$.

7.1. Definition. Let $f \in \operatorname{Diff}(M)$ where $M$ is compact. Then $f$ is $\Omega$-hyperbolic iff $\Omega(f)$ is a hyperbolic invariant set for $f ; f$ is $P$-hyperbolic iff each periodic orbit of $f$ is hyperbolic; $f$ satisfies axiom A iff $f$ is $\Omega$-hyperbolic and $\bar{P}(f)=\Omega(f) ; f$ satisfies weak axiom A iff $f$ is $P$-hyperbolic and $\bar{P}(f)=\Omega(f)$.

(The definition of axiom A is due to Smale [51].)

Two reasonable (and related) conjectures are:

(i) If $f$ is $\Omega$-hyperbolic, then $\bar{P}(f)=\Omega(f)$.

(ii) If $f$ is Anosov, then $\Omega(f)=M$.

Some progress on these questions has been made by Newhouse and Palis ([27], [28], and [29]).

The following theorem is due to the combined work of Kupka, Smale, Peixoto, and Pugh. (See [3], [22], [35], [37], [38], and [50].)

7.2. THEOREM. Weak axiom A is a generic property ${ }^{12}$ on $\operatorname{Diff}(M)$.

${ }^{12}$ A property is called generic on $\operatorname{Diff}(M)$ iff it is true on a residual subset of $\operatorname{Diff}(M)$. A residual subset is a countable intersection of open dense sets and is thus dense (Baire category theorem). Thus a generic property holds on a dense subset of $\operatorname{Diff}(M)$. 
7.3. Corollary. ${ }^{13} A$ structurally stable diffeomorphism satisfies weak axiom A.

In contrast to 7.2 we have

7.4. ExAmple [0]. There is an open subset $N$ of $\operatorname{Diff}(M)$ where $M=T^{2} \times S^{2}$ such that no $f \in N$ satisfies axiom A.

Note that when $\bar{P}=\Omega, \Omega$-hyperbolicity is a kind of uniform $P$-hyperbolicity; it says that the hyperbolic structures on the periodic orbits extend continuously to $\Omega$. This prompts the following

7.5. CONJECTURE. A structurally stable diffeomorphism satisfies axiom A.

7.6. Definition. Let $f \in \operatorname{Diff}(M)$. The essential spectrum of $f$ is the closure of the set $U \operatorname{spec}(\operatorname{ad}(f, \Lambda))$ where the union is over all periodic orbits $\Lambda$ of $f$ and $\operatorname{spec}(\operatorname{ad}(f, \Lambda))$ denotes the spectrum (in this case, the set of eigenvalues) of the finite dimensional linear operator $\operatorname{ad}(f, \Lambda): C^{0}(T M \mid \Lambda)$ $\rightarrow C^{0}(T M \mid \Lambda)$.

It is not hard to see that (when $M$ is compact) the essential spectrum of $f$ is a subset of the spectrum of $\operatorname{ad}(f, \bar{P}(f))$.

7.7. Conjecture. ${ }^{14}$ The essential spectrum of $f$ is the spectrum of $\operatorname{ad}(f, \bar{P}(f))$.

7.8. TheOREM (FRANKS [8]). If $f$ is structurally stable, then the essential spectrum of $f$ contains no complex number of modius one.

Thus 7.7 (for structurally stable $f$ ) together with 7.8 and 7.3 would imply 7.5 .

8. Transversality. Let $f \in \operatorname{Diff}(M)$ where $M$ is compact. We give $M$ a Riemannian metric which determines a metric $d$ on $M$, but all the definitions of this section are easily seen to be independent of the metric (by compactness arguments).

Define $E^{ \pm}(f)=E^{ \pm} \subseteq T M$ by

$$
\begin{aligned}
& E^{+}=\left\{\dot{x} \in T M \mid \lim _{n \rightarrow \infty}\left\|T f^{n} \dot{x}\right\|=0\right\}, \\
& E^{-}=\left\{\dot{x} \in T M \mid \lim _{n \rightarrow \infty}\left\|T f^{-n} \dot{x}\right\|=0\right\} ;
\end{aligned}
$$

and for $x \in M$ let $E_{x}^{ \pm}=E^{ \pm} \cap T_{x} M$. In case $\Lambda$ is a hyperbolic invariant set the notation $E^{ \pm} \mid \Lambda$ agrees with that of Theorem 6.4 but, in general, $E^{+}$and $E^{-}$are not subbundles of $T M$ (see Example 1.8). Note, however,

137.3 is an easy but not obvious consequence of 7.2. The proof requires the stable-unstable manifold theory described in $\S 8$. It is easy to see that 7.2 implies that $\bar{P}=\Omega$ for a structurally stable diffeomorphism as this property is topologically invariant. $P$-hyperbolicity is not a topologically invariant property.

${ }^{14}$ Due independently to Abraham and Shub. 
that $E_{x}^{+}$and $E_{x}^{-}$are vector subspaces of $T_{x} M$ for $x \in M$.

8.1. Definition. Let $f \in \operatorname{Diff}(M)$ where $M$ is compact. Then $f$ satisfies the strong transversality condition iff $T_{x} M=E_{x}^{+}+E_{x}^{-}$for all $x \in M$ where the sum need not be direct.

A motivation of this definition requires a brief excursion into stable manifold theory. For $\Lambda \subseteq M$ (not necessarily invariant) define $W^{ \pm}(\Lambda, f)$ $=W^{ \pm}(\Lambda) \subseteq M$ by

$$
\begin{aligned}
& W^{+}(\Lambda)=\left\{y \in M \mid \lim _{n \rightarrow \infty} d\left(f^{n}(y), f^{n}(\Lambda)\right)=0\right\}, \\
& W^{-}(\Lambda)=\left\{y \in M \mid \lim _{n \rightarrow \infty} d\left(f^{-n}(y), f^{-n}(\Lambda)\right)=0\right\} .
\end{aligned}
$$

Then $W^{+}(\Lambda)$ is called the in-set of $\Lambda$ (for $f$ ) and $W^{-}(\Lambda)$ is called the out-set of $\Lambda$. When $\Lambda$ is a point, $\Lambda=\{x\}$, we denote $W^{ \pm}(\Lambda)$ by $W^{ \pm}(x)$. Traditionally, $W^{+}(x)$ and $W^{-}(x)$ are called respectively the stable and unstable manifolds of $x$ and are denoted by $W^{s}(x)$ and $W^{u}(x)$. The simpler terminology is due to Zeeman.

Note that the sets $W^{+}(x)$ partition $M: x \in W^{+}(x)$ and $W^{+}(x) \cap W^{+}(y)$ $\neq \varnothing$ if and only if $W^{+}(x)=W^{+}(y)$. Similarly for $W^{-}$.

The following is a generalization of a classical theorem (see [13], [18], and [44] for example) in case $\Lambda$ is a fixed point of $f$ :

8.2. THEOREM [16]. Let $\Lambda$ be a compact hyperbolic invariant set of $f \in \operatorname{Diff}(M)$ and let $x \in \Lambda$. Suppose $f$ is $C^{r}(r \geqq 1)$. Then $W^{ \pm}(x)$ are $C^{r}$ injectively immersed cells and for $y \in W^{ \pm}(x), E_{y}^{ \pm}=T_{y} W^{ \pm}(x)$.

8.3. Definition. Let $f \in \operatorname{Diff}(M)$ and $\Lambda \subseteq M$. Then $\Lambda$ is in-phase (for $f$ ) iff $W^{ \pm}(\Lambda)=\bigcup_{x \in \Lambda} W^{ \pm}(x)$.

A hyperbolic invariant set need not be in-phase (see [17]), but

8.4. Theorem [17]. If $f \in \operatorname{Diff}(M)$ satisfies axiom $\mathrm{A}$, then $\Omega(f)$ is in-phase.

Now, it is always true that $W^{+}(\Omega)=W^{-}(\Omega)=M$ when $M$ is compact. Hence by 8.2 and 8.4 we see that if $f$ satisfies axiom $\mathrm{A}$, then $f$ satisfies the strong transversality condition iff for all $x, y \in \Omega(f), W^{+}(x)$ and $W^{-}(y)$ intersect transversally (see [3]).

A diffeomorphism $f \in \operatorname{Diff}(M)$ satisfies the weak transversality condition iff $W^{+}(x)$ and $W^{-}(y)$ intersect transversally for all (hyperbolic) periodic points $x$ and $y$. According to the theorem of Kupka-Smale, the weak transversality condition is a generic property (see [3], [22], [35], and [50]). In contrast, Smale [49] gives an example of an open set $N \subseteq \operatorname{Diff}(M)$ such that every $f \in N$ satisfies axiom A and no $f \in N$ satisfies the strong transversality condition. Also no $f \in N$ is structurally stable. Shub [48] proves, however, that the structurally stable systems are dense in the $C^{0}$-topology, 
The following theorem ties together a great many diverse ideas:

8.5. Theorem. Let $f \in \operatorname{Diff}(M)$ where $M$ is compact and suppose $f$ satisfies weak axiom A. Then $1-\operatorname{ad}(f)$ is surjective if and only if $f$ satisfies axiom $\mathrm{A}$ and the strong transversality condition.

Proof. The "if" direction is proved in [45]; we shall prove "only if". Assume $1-\operatorname{ad}(f)$ is surjective.

By the section extension theorem, $1-\operatorname{ad}(f, \Omega)$ is surjective. By weak axiom $\mathrm{A}, 1-\operatorname{ad}(f, \Omega)$ is injective. Thus $1-\operatorname{ad}(f, \Omega)$ is bijective and by a version of 6.2 (see [9]) it follows that $\operatorname{ad}(f, \Omega)$ is hyperbolic and hence that $f$ satisfies axiom $\mathrm{A}$.

Suppose $f$ does not satisfy the strong transversality condition; we will prove a contradiction. Then there exists $x \in M$ with

$$
E_{x}^{+}+E_{x}^{-} \neq T_{x} M \text {. }
$$

As $f$ is $\Omega$-hyperbolic, $x \notin \Omega(f)$. Thus there exists a neighborhood $U$ of $x$ with $f^{n}(U) \cap U=\varnothing$ for all $n \neq 0$. Choose $\zeta \in C^{0}(T M)$ supported in $U$ with

$$
\zeta(x) \notin E_{x}^{+}+E_{x}^{-}
$$

(possible by (1)). Then $\zeta\left(f^{n}(x)\right)=0$ for $n \neq 0$. Note also that $\zeta \mid \Omega \equiv 0$.

As $1-\operatorname{ad}(f)$ is surjective, there exists $\eta \in C^{0}(T M)$ with

$$
(1-\operatorname{ad}(f)) \eta=\zeta \text {. }
$$

Then

$$
\left(1-\operatorname{ad}(f)^{n+1}\right) \eta=\sum_{k=0}^{n} \operatorname{ad}(f)^{k} \zeta,
$$

and as $\operatorname{ad}(f)^{k} \zeta(x)=0$ for $k \neq 0$ we obtain $\eta(x)-T f^{n+1} \eta\left(f^{-n-1}(x)\right)$ $=\zeta(x)$ or

$$
T f^{-n-1}(\eta(x)-\zeta(x))=\eta\left(f^{-n-1}(x)\right) .
$$

As $n \rightarrow \infty, f^{-n-1}(x) \rightarrow \Omega$. Restricting (3) to $\Omega$ and using $\Omega$-hyperbolicity and the fact that $\zeta \mid \Omega \equiv 0$ gives that $\eta \mid \Omega \equiv 0$. Thus from (4) we obtain

$$
\eta(x)-\zeta(x) \in E_{x}^{-} .
$$

Similarly,

$$
\left(1-\operatorname{ad}(f)^{-n}\right) \eta=-\sum_{k=1}^{n} \operatorname{ad}(f)^{-k \zeta}
$$

so $\eta(x)-T f^{-n} \eta\left(f^{n}(x)\right)=0$, so $T f^{n} \eta(x)=\eta\left(f^{n}(x)\right) \rightarrow 0$ as $n \rightarrow \infty$, so

$$
\eta(x) \in E_{x}^{+} \text {. }
$$

Substract (5) from (6) to obtain $\zeta(x) \in E_{x}^{+}+E_{x}^{-}$, which contradicts (2).

9. The characterization theorem. Smale ([53]; see also [34]) has 
proposed the following solution for Problem I of $\S 1 \mathrm{E}$ :

9.1. ConjeCture. A diffeomorphism is structurally stable if and only if it satisfies axiom $\mathrm{A}$ and the strong transversality condition.

This conjecture is proved in [34] for the special case where $\Omega$ is finite. The general case is as yet unsolved, the only obstacle being conjecture 7.5. For the case of absolute structural stability, the situation is better:

9.2. THEOREM. Let $f \in \operatorname{Diff}(M)$ where $M$ is compact and suppose $e^{15}$ that $f$ is $C^{2}$. Then the following are equivalent:

I. $f$ satisfies axiom $\mathrm{A}$ and the strong transversality condition;

II. $f$ is infinitesimally stable;

III. $f$ is absolutely structurally stable;

IV. $f$ is structurally stable and $1-\operatorname{ad}(f)$ is surjective.

I implies II implies III is proved in [45] (except that III is replaced by strong structural stability); the introduction to [45] gives a sketch of the proof. Refer also to $\$ 4$ for the idea of II implies III. III implies IV follows from 4.6 and IV implies I follows from 7.3 and 8.5.

Condition IV seems to say that " $f$ is structurally stable and this can be proved using the implicit function theorem." Thus if Conjecture 9.1 is false one will have to construct a structurally stable diffeomorphism and prove its stability by an argument more general than the contraction mapping principle.

Clearly Conjecture 9.1 follows from Conjecture 7.5 and Theorem 9.2. As explained in $\$ 7$, Conjecture 7.5 follows from Conjecture 7.7; thus 9.1 looks very plausible and hence also the equivalence of the various concepts of structural stability $(1.6,1.10$ and 1.12).

I am interested in the question of whether $1-\operatorname{ad}(f)$ surjective implies axiom A. An affirmative answer would strengthen Theorem 8.5 and also prove a version of Conjecture 4.4. It would enable us to "drop to dots" in the definition of infinitesimal stability (see 4.7).

Finally I should like to say that the classification problem (Problem II of $\S 1 \mathrm{E}$ ) for structurally stable diffeomorphisms does not appear to me to be hopeless. The work of Bowen and Williams cited in the bibliography goes a long way towards describing $\Omega(f)$ for an axiom A diffeomorphism. The work of Franks, Hirsch, and Shub (among others) bears strongly on Problem II for Anosov diffeomorphisms (see [10], [11], [15], [46], and [47]). The labeled diagrams of Smale [51] suggest a first step in attacking Problem II in the case of structurally stable systems with finite nonwandering set (Morse-Smale systems); see Palis [33].

\footnotetext{
15 As pointed out in [45] this condition can probably be dropped; in fact, Wellington Melo [59] has already succeeded in doing this in case $M$ is two-dimensional.
} 


\section{BIBLIOGRAPHY}

0. R. Abraham and S. Smale, Nongenericity of $\Omega$-stability, Proc. Sympos. Pure Math., vol. 14, Amer. Math. Soc., Providence, R. I., 1970, pp. 5-8. MR 42 \# 6867; See also: S. Newhouse, Nondensity of axiom $A(a)$ on $S^{2}$, ibid., pp. 191-202. MR 43 \#2742.

1. R. Abraham, Predictions for the future of differential equations, Sympos. on Differential Equations and Dynamical Systems, Lecture Notes in Math., no. 206, Springer-Verlag, Berlin and New York, 1971.

2. R. Abraham and J. Marsden, Foundations of mechanics, Benjamin, New York, 1967. MR 36 \# 3527.

3. R. Abraham and J. Robbin, Transversal mappings and flows, Benjamin, New York, 1967. MR 39 \# 2181.

4. A. Andronov and L. Pontrjagin, Structurally stable systems, Dokl. Akad. Nauk SSSR 14 (1937), 247-250. (Russian)

5. D. V. Anosov, Geodesic flows on closed Riemannian manifolds of negative curvature, Trudy Mat. Inst. Steklov. 90 (1967) = Proc. Steklov Inst. Math. 90 (1967), MR 36 \# 7157. 6. R. Bowen, Markov partitions for axiom A diffeomorphisms, Amer. J. Math. 92 (1970), 725-747. MR 43 \# 2740.

7. - Markov partitions and minimal sets for axiom A diffeomorphisms, Amer. J. Math. 92 (1970), 907-918. MR 43 \# 2739.

8. J. Franks, Necessary conditions for stability of diffeomorphisms, Trans. Amer. Math. Soc. 158 (1971), 301-308.

9. - Differentiable $\Omega$-stability, Topology 11 (1972), 107-112.

10. _ Anosov diffeomorphisms, Proc. Sympos. Pure Math., vol. 14, Amer. Math.

Soc., Providence, R. I., 1970, pp. 61-93. MR 42 \# 6871.

11. 125. MR 40 \# 6567.

12. J. Guckenheimer, Absolutely $\Omega$-stable diffeomorphisms (to appear).

13. P. Hartman, Ordinary differential equations, Wiley, New York, 1964. MR 30 \# 1270.

14. - A lemma in the theory of structural stability of differential equations, Proc.

Amer. Math. Soc. 11 (1960), 610-620. MR 22 \# 12279.

15. M. Hirsch, Expanding maps and transformation groups, Proc. Sympos. Pure Math., vol. 14, Amer. Math. Soc., Providence, R.I., 1970, pp. 125-131.

16. M. Hirsch and C. Pugh, Stable manifolds and hyperbolic sets, Proc. Sympos. Pure

Math., vol. 14, Amer. Math. Soc., Providence, R.I., 1970, pp. 133-163. MR 42 \#6872.

17. M. Hirsch, J. Palis, C. Pugh and M. Shub, Neighbourhoods of hyperbolic sets, Invent.

Math. 9 (1969/70), 121-134. MR 41 \# 7232.

18. M. Irwin, On the stable manifold theorem, Bull. London Math. Soc. 2 (1970), 196-

198. MR 42 \# 6873. See also: M. Irwin, On the smoothness of the composition map, Quart.

J. Math. Oxford (2), 23 (1972), 113-133.

19. S. Kobayashi and K. Nomizu, Foundations of differential geometry. Vol. 1, Interscience, New York. 1963. MR 27 \# 2945.

20. R. Kirby and L. Siebenmann, On the triangulation of manifolds and the Hauptvermutung, Bull. Amer. Math. Soc. 75 (1969), 742-749. MR 39 \#3500.

21. N. H. Kuiper and J. Robbin, Topological classification of linear automorphisms (to appear).

22. J. Kupka, Contribution à la théorie des champs génériques, Contributions to Differential Equations 2 (1963), 457-484.

23. L. H. Loomis, An introduction to abstract harmonic analysis, Van Nostrand, Princeton, N.J., 1953, p. 17. MR 14, 883.

24. J. N. Mather, Characterization of Anosov diffeomorphisms, Nederl. Akad. Wetensch. Proc. Ser. A 71 = Indag. Math. 30 (1968), 479-483. MR 40 \# 2129.

25. J. K. Moser, On a theorem of Anosov, J. Differential Equations 5 (1969), 411-440. MR 38 \#6633.

26. J. R. Munkres, Elementary differential topology, Ann. of Math. Studies, no. 54, Princeton Univ. Press, Princeton, N.J., 1963. MR 29 \#623.

27. S. Newhouse, Hyperbolic limit sets, Trans. Amer. Math. Soc. 167 (1972), 125-150. 28. _- On codimension one Anosov diffeomorphisms, Amer. J. Math. 92 (1970), 761-770. MR 43 \# 2741 .

29. S. Newhouse and J. Palis, Hyperbolic non-wandering sets on two-dimensional manifolds, Proc. Internat. Sympos. on Dynamical Systems held in Salvador, Bahia, Brazil, 1971 (to appear).

30. Z. Nitecki, On semi-stability for diffeomorphisms, Invent. Math. 14 (1971), 83-123.

31. R. S. Palais, Foundations of global non-linear analysis, Benjamin, New York, 1968. 
MR 40 \# 2130. See also: J. Eells, A setting for global analysis, Bull. Amer. Math. Soc. 72 (1966), 751-807.

32. J. Palis, On the local structure of hyperbolic points in Banach spaces, An. Acad. Brasil. Ci. 40 (1968), 263-266. MR 39 \# 7635.

33. - On Morse-Smale dynamical systems, Topology 8 (1969), 385-404. MR 39 \# 7620.

34. J. Palis and S. Smale, Structural stability theorems, Proc. Sympos. Pure Math., vol. 14, Amer. Math. Soc., Providence, R.I., 1970, pp. 223-231. MR 42 \# 2505.

35. M. M. Peixoto, On an approximation theorem of Kupka and Smale, J. Differential Equations 3 (1967), 214-227. MR 35 \# 499.

36. _ Structural stability on two-dimensional manifolds, Topology 1 (1962), 101120. MR 26 \# 426

37. C. Pugh, The closing lemma, Amer. J. Math. 89 (1967), 956-1009. MR 37 \# 2256. 38. - An improved closing lemma and a general density theorem, Amer. J. Math. 89 (1967), 1010-1021. MR 37 \# 2257.

39. - On a theorem of P. Hartman, Amer. J. Math. 91 (1969), 363-367. MR 41 \# 2183.

40. G. de Rham, Reidemeister's torsion invariant and rotations of $S^{n}$, Differential Analysis, Bombay Colloq., 1964, Oxford Univ. Press, London, 1964, pp. 27-36. MR 32 \# 8355.

41. G. de Rham, S. Maumary and M. A. Kervaire, Torsion et type simple d'homotopie, Lecture Notes in Math., no. 48, Springer-Verlag, Berlin and New York, 1967. MR 36 \# 5943.

42. S. Robertson, Equivalence of dynamical systems, Sympos. on Differential Equations and Dynamical Systems, Lecture Notes in Math., no. 206, Springer-Verlag, Berlin and New York, 1971

43. C. Robinson, Generic properties of conservative systems. I, II, Amer. J. Math. 92 (1970), 562-603, 897-906.

44. J. Robbin, Stable manifolds for semi-hyperbolic fixed points, Illinois J. Math. 15 (1971), 595-609.

45. - A structural stability theorem, Ann. of Math. (2) 94 (1971), 447-493.

46. M. Shub, Expanding maps, Proc. Sympos. Pure Math., vol. 14, Amer. Math. Soc., Providence, R.I., 1970, pp. 273-276. MR 42 \# 1158.

47. — Endomorphisms of compact differentiable manifolds, Amer. J. Math. 91 (1969), 175-199. MR 39 \# 2169.

48. - Structurally stable diffeomorphisms are dense (to appear).

49. S. Smale, Structurally stable systems are not dense, Amer. J. Math. 88 (1966), 491496. MR 33 \# 4911

50. - Stable manifolds for differential equations and diffeomorphisms, Ann. Scuola Norm. Sup. Pisa (3) 17 (1963), 97-116. MR 29 \# 2818b.

51. — Differentiable dynamical systems, Bull. Amer. Math. Soc. 73 (1967), 747817. MR 37 \# 3598.

52. - Global stability questions in dynamical systems, Lectures in Modern Analysis and Applications. I, Springer, Berlin, 1969, pp. 150-158. MR 40 \# 2131.

53. R. G. Swan, Vector bundles and projective modules, Trans. Amer. Math. Soc. 105 (1962), 264-277. MR 26 \# 785.

54. P. Walters, Anosov diffeomorphisms are topologically stable, Topology 9 (1970), 71-78. MR 40 \# 8069.

55. R. F. Williams, One-dimensional non-wandering sets, Topology 6 (1967), 473-487. MR 36 \# 897.

56. ㄴ. Classification of one dimensional attractors, Proc. Sympos. Pure Math., vol. 14, Amer. Math. Soc., Providence, R.I., 1970, pp. 341-361. MR 42 \# 1134.

57. - - Expanding attractors, Northwestern University, Evanston, Ill., preprint (to appear)

58. - Classification of subshifts of finite type, Northwestern University, Evanston, Ill., preprint (to appear).

59. W. Melo, Structural stability on two-dimensional manifolds, Thesis, Instituto de Matématica Pura e Aplicada, Rio de Janeiro, Brazil, 1972; Proc. Internat. Sympos. on Dynamical Systems, Salvador, Brazil, Academic Press, New York (to appear).

Current address: Department of Mathematics, University of Wisconsin, Madison, Wisconsin 53706. 